\title{
T-STRESS OF CONCRETE UNDER FOUR-POINT BENDING
}

\author{
ZHAO YANHUA ${ }^{*}$, LIU JIN ${ }^{\dagger}$ \\ State Key Laboratory of Coastal and Offshore Engineering \\ Dalian University of Technology, Dalian P.R.China \\ e-mail: yanhuazh@dlut.edu.cn \\ ${ }^{\dagger}$ State Key Laboratory of Coastal and Offshore Engineering \\ Dalian University of Technology, Dalian P.R.China \\ e-mail:814969159@qq.com
}

Key words: Fracture Mechanics, Stress Intensity Factor, $T$-stress, Four-point Bending

\begin{abstract}
Mixed mode stress-intensity factors (SIFs) and the $T$-stress for different geometries of four-point bending specimens were calculated by means of finite element analysis through a software suite Abaqus, where the influence of the loading point and crack location were considered. The fracture properties, $K_{\mathrm{I}}, K_{\mathrm{II}}$ and $T$-stress expressions for four-point bending were constructed based on pure bending and pure shear by means of finite element calculation. The results reveal that considerable values of the $T$-stress exist with different combinations of $K_{\mathrm{I}}$ and $K_{\mathrm{II}}$, and the stress biaxiality factor $B$ varies, including its magnitude and sign, along with the change in geometry size.
\end{abstract}

\section{INTRODUCTION}

As the constant term of the infinite Williams series expansion for stress component parallel to the crack flank ${ }^{[1]}, T$ stress is believed to significantly influence the stress and strain fields around the crack tip, and thus the failure of fracture. Recently, the authors have explored the $T$-stress influence on the fracture toughness $K_{\text {Ic }}$ under mode I loading condition and concluded that $T$-stress values are so small compared to the singular term thus fracture toughness $K_{\text {Ic }}$ deduced from the conventional ways are reliable ${ }^{[2]}$. As an extension, the authors attempt to study the $T$ stress influence on the fracture toughness of concrete under mixed mode loading. But before that, complete expressions for fracture parameters $\left(K_{\mathrm{I}}, K_{\mathrm{II}}\right.$ and $T$-stress) at the crack tip should be necessary. In the following sections, the modified MTS criterion with $T$ stress involved is first reviewed. Then expressions for fracture parameters $\left(K_{\mathrm{I}}, K_{\mathrm{II}}\right.$ and
$T$-stress) at the crack tip of four point bending beams are constructed from finite element analysis.

\section{MODIFIED MTS CRITERION FOR I/II MIXED MODE FRACTURE}

According to the modified MTS criterion ${ }^{[3]}$, the direction of crack initiation $\theta_{0}$ is obtained through

$K_{\mathrm{I}} \sin \theta_{0}+K_{\mathrm{II}}\left(3 \cos \theta_{0}-1\right)=\frac{16}{3} T \sqrt{2 \pi r_{\mathrm{c}}} \cos \theta_{0} \sin \frac{\theta_{0}}{2}$

and a parametric equation of initiation cracking curve in $K_{\mathrm{I}}-K_{\text {II }}$ plane is

$$
\left\{\begin{array}{l}
\frac{K_{\mathrm{I}}}{K_{\mathrm{Ic}}}=\gamma \frac{K_{\mathrm{II}}}{K_{\mathrm{Ic}}} \\
\frac{K_{\mathrm{II}}}{K_{\mathrm{Ic}}}=\frac{1}{\cos \frac{\theta_{0}}{2}\left(\gamma \cos ^{2} \frac{\theta_{0}}{2}-\frac{3}{2} \sin \theta_{0}\right)+B \sqrt{\frac{2 \pi r_{\mathrm{c}}}{a}} \sqrt{1+\gamma^{2}} \sin ^{2} \theta_{0}}
\end{array}\right.
$$


where $a$ represents crack length, and $\gamma=K_{\mathrm{I}} / K_{\mathrm{II}}$ is the ratio of SIFs between mode I and mode II; $B$ is the normalized $T$-stress called stress biaxiality factor in [4]

$$
B=\frac{T \sqrt{\pi a}}{K_{\text {eff }}}
$$

where the effective SIF $K_{\text {eff }}=\sqrt{K_{\mathrm{I}}^{2}+K_{\mathrm{II}}^{2}}$ is the equivalent SIF for a crack under I/II mixed mode loading.

When the conventional MTS with $B$ in Eq.(2) equal to zero is used to address initiation cracking problems, discrepancy is often observed, especially for pure mode II and I/II mixed mode fracture, and researchers begin to realize the significance of $T$-stress. In the following sections, $T$-stress influence on I/II mixed mode fracture in concrete is studied, but before that, an introduction to the specimen we used for I/II mixed mode fracture is more than necessary.

\section{CALCULATION OF $K_{\mathrm{I}}, K_{\mathrm{II}}$ AND $T$ AT THE CRACK TIP UNDER FOUR-POINT BENDING}

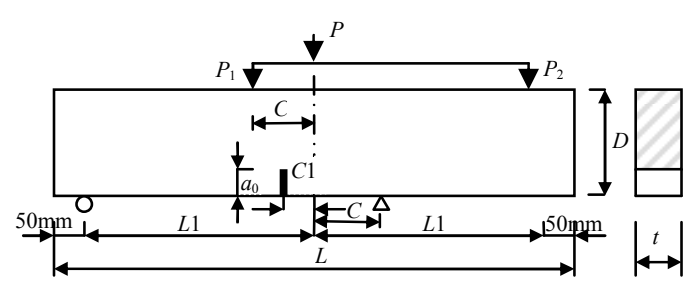

(a) Schematic representation of four-point bending beam

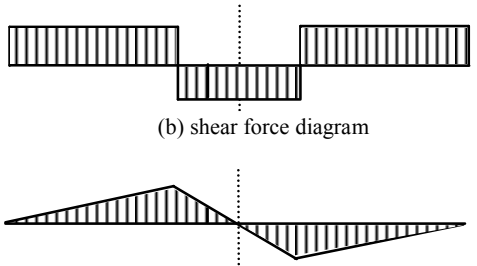

(c) bending moment diagram

Fig.1 Geometry of the four-point bending specimen

The four-point bending beam (c.f. Fig.1) is a rectangular-cross beam with an edge crack of length $a$. The beam is subjected to four concentrated loads which are anti-symmetric relative to the middle line of the specimen, and $C 1$ is the crack distance from the specimen middle. The four-point bending beam is nowadays among the frequently used configurations to study I/II mixed mode fracture for concrete ${ }^{[5]}$ due to its simple geometry and loading set up. Particularly, it was once believed that pure mode I and pure mode II could be easily created just by setting $C=0, C 1=0$ and $C 1=0$, respectively.

For a four-point bending notched beam shown in Fig.1, mixed mode SIFs, even $T$ stress were investigated for some specific cases, such as $C 1=0$ or small values of $C 1 / C^{[6,}$ ${ }^{7,8]}$. From Fig. $1, C 1 / C$ should take values from 0 to 1 so that a wide range of $K_{\mathrm{I}} / K_{\text {II }}$ values can be covered for a complete cracking initiation curve. However, no exact solutions regarding fracture properties $\left(K_{\mathrm{I}}, K_{\mathrm{II}}\right.$ and $\left.T\right)$ for this case are reported in the literature. Therefore, the finite-element code Abaqus was conducted to get these fracture properties for 891 different geometries and loading conditions, which included 9 cases of different loading distance from the centerline of the specimen, $C / D=0.2$ 1 with an interval of $0.1 ; 11$ cases for different crack location for every $C / D, C 1 / C=0-1$ with 0.1 as increment; and 9 cases for different crack length for every $C 1 / C, a / D=0.1-0.9$ with an increment of 0.1 . The dimensions of the four-point bend beam in the modeling were selected as: $L=900 \mathrm{~mm}, \quad L 1=400 \mathrm{~mm}$, $D=200 \mathrm{~mm}, t=40 \mathrm{~mm}$. The magnitude of the applied force was $P=1000$ N. An average 7000 number of elements and 20000 of nodes were used for the discretization, where main element type CPS8, CPS6 at the crack tip, Young's modulus E=30MPa and Poisson's ratio $\mu=0.2$ were considered to perform model simulation. Interaction integral method readily available in the code Abaqus was employed to extract $K_{\mathrm{I}}, K_{\mathrm{II}}$ and $T$-stress at the pre-crack tip. The background is not described here, and readers who are interested can find the relevant information in Abaqus's help file.

As far as four-point bend beam shown in Fig. 1 is concerned, $K_{\text {II }}\left(K_{\text {II }}\right.$ is defined per unit thickness) at the crack tip can be expressed from dimensional considerations as

$$
K_{\mathrm{II}}=K_{\mathrm{II}}\left(\frac{C}{D}, \frac{C 1}{C}, \frac{a}{D}\right)=\beta_{\mathrm{II}} \frac{Q}{D^{1 / 2}} \frac{\alpha^{3 / 2}}{(1-\alpha)^{1 / 2}} F_{\mathrm{II}}(\alpha)
$$


where $\alpha=a / D$ is the relative crack length; $Q$ is the shear force between the inner loading points and it is related to the force $P$ by

$$
Q=P \frac{L 1-C}{L 1+C}
$$

$\beta_{\mathrm{II}}$ is the correction factor for $K_{\mathrm{II}}$ when compared to the situation of a pure shear force $Q$ acting on an infinitely long cracked specimen, and it is introduced to allow for the influence of loading point and the crack location. Non-dimensional function $F_{\text {II }}(\alpha)$ in Eq.(4) was obtained by fitting the numerical results in [6]

$$
F_{\text {II }}(\alpha)=7.264-9.37 \alpha+2.74 \alpha^{2}+1.87 \alpha^{3}-1.04 \alpha^{4}
$$

The above polynomial representation is believed to be accurate to within $1 \%$ over the entire range of $\alpha$.

Similarly, $K_{\mathrm{I}}$ at the crack tip is expected to build on the reference of pure moment case, but one thing should be noted: when $C_{1}=0$, only shear force is acting on the crack plane, i.e., $M=0$, but $K_{\mathrm{I}}$ is not precisely zero. Therefore, $K_{\mathrm{I}}$ for the case shown in Fig.1 is assumed to take the following form

$$
K_{\mathrm{I}}=K_{\mathrm{I}}\left(\frac{C}{D}, \frac{C 1}{C}, \frac{a}{D}\right)=\beta_{\mathrm{I}} \frac{6 Q(C 1+C 2)}{D^{2}} \sqrt{\pi a} F_{\mathrm{I}}(\alpha)
$$

where $C 2$ is a virtual distance so that the pure moment $M=Q C 2$ could produce $K_{\mathrm{I}}$ equivalently to a center crack in Fig. 1 , and $\beta_{\mathrm{I}}$ is the correction factor for $K_{\mathrm{I}}$ to account for the effect of the crack location. Function $F_{\mathrm{I}}(\alpha)$ in Eq.(7) was defined by Tada et al. as ${ }^{[9]}$

$$
F_{\mathrm{I}}(\alpha)=\sqrt{\frac{2}{\pi \alpha} \tan \frac{\pi \alpha}{2}} \frac{0.923+0.199\left(1-\sin \frac{\pi \alpha}{2}\right)^{4}}{\cos \frac{\pi \alpha}{2}}
$$

The above formula has better than $0.5 \%$ for any $\alpha$.

Figs. 2 and 3 depicts some numerical results obtained for $\beta_{\mathrm{I}}, \beta_{\mathrm{II}}$ and $B$ for $C / D=0.3$ and $C / D=0.8$ as a function of $\alpha$ for different values of $C 1 / D$.

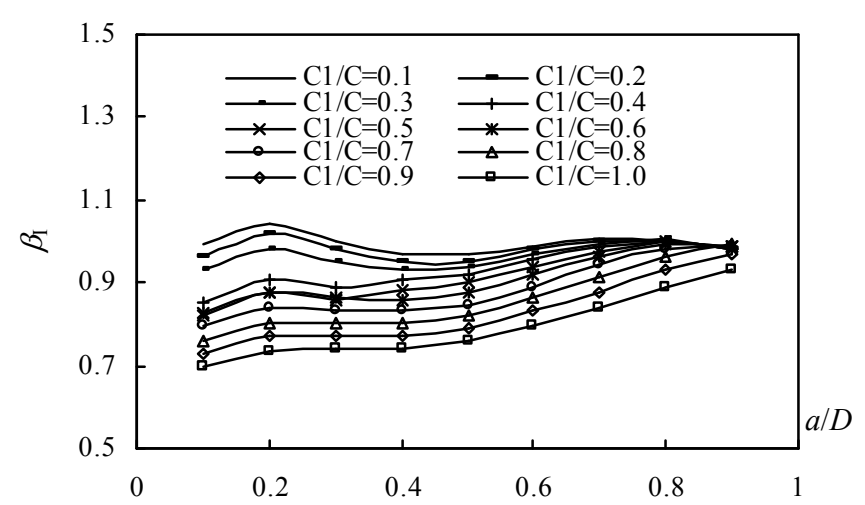

(a) $\beta_{\mathrm{I}}$

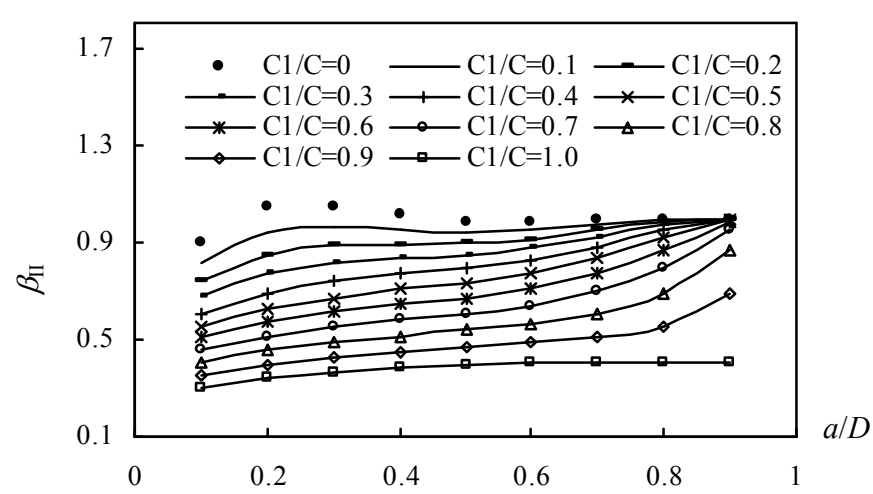

(b) $\beta_{\mathrm{II}}$

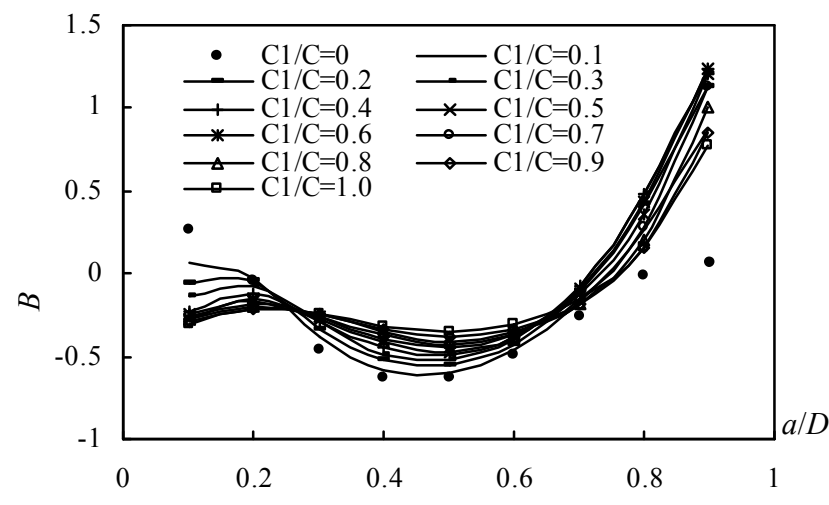

(c) $B$

Fig. $2 \beta_{\mathrm{I}}, \beta_{\mathrm{II}}$ and $B$ for $\mathrm{C} / \mathrm{D}=0.3$

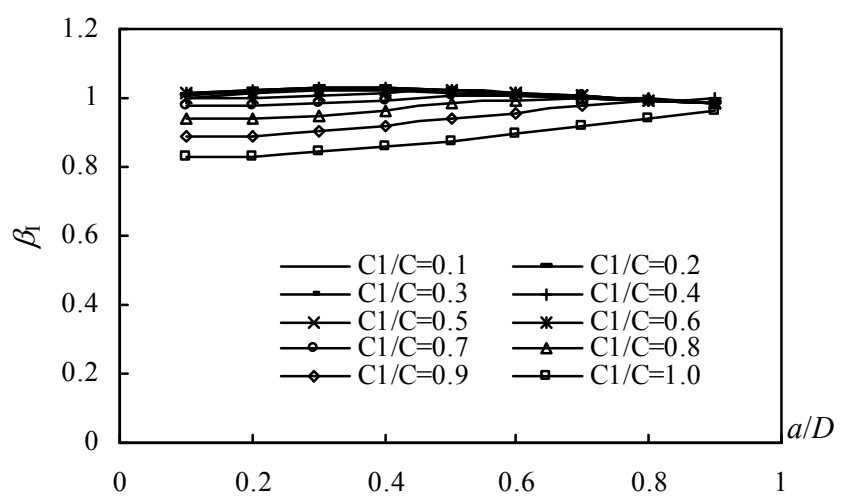

(a) $\beta_{\mathrm{I}}$ 


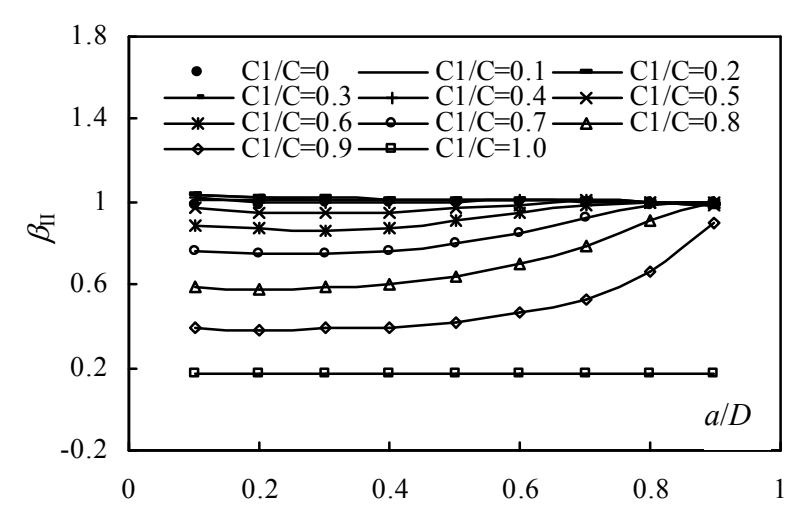

(b) $\beta_{\text {II }}$

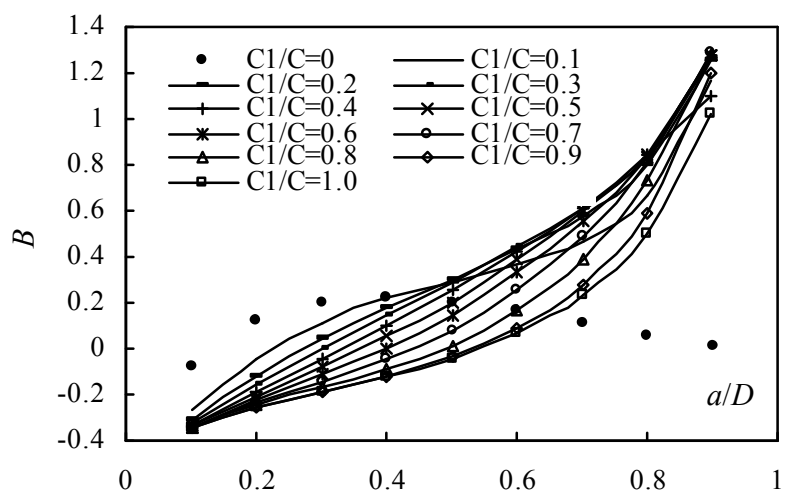

(c) $B$

Fig. $3 \beta_{\mathrm{I}}, \beta_{\mathrm{II}}$ and $B$ for $\mathrm{C} / \mathrm{D}=0.8$

The finite element analysis indicates the crack tip parameters $K_{\mathrm{I}}, K_{\mathrm{II}}$ and $T$-stress are strongly dependent on the geometry and loading configuration. Specifically, the crack location and loading distance both exert great influence on mode I and mode II fracture, which are reflected by the correction factor $\beta_{\mathrm{I}}$ and $\beta_{\mathrm{II}}$ in Eq.(7) and Eq.(4). A wide range of combination of $K_{\mathrm{I}}$ and $K_{\mathrm{II}}$ are obtained by changing the loading point $C$ and crack location $C 1$. When $C=C 1=0$, pure mode I fracture can be obtained. Nevertheless, $C 1=0$ together with $C \neq 0$ is not able to provide pure II fracture mode since both $K_{1}$ and $T$-stress appear in the crack-tip parameters. Furthermore, $K_{1}$ is negative for small value $C / D=0.2$, technically meaning the crack flanks tend to close. However, it is required that $K_{\mathrm{I}}>0$, or $K_{\mathrm{I}}=0$ at most for an opening crack. Thus, $K_{\mathrm{I}}$ is supposed to be zero for this occasion, which will be demonstrated in the later section. Even though $K_{\mathrm{I}}=0$ for $C 1=0$ and small value $C / D=0.2$, pure mode II fracture is not achieved due to the existence of $T$-stress. Pure II mode fracture can hopefully be achieved by imposing load far sufficiently from the crack plane. [6] pointed out when $C / D$ is greater than $1.4, \beta_{\mathrm{II}}$ in Eq.(4) is nearly to 1 , meaning a valid pure shear condition, and [7] suggested that $C / D$ should be 1.5 or greater to eliminate the effects of $K_{\mathrm{I}}$ and $T$-stress for pure mode II fracture.

The biaxiality ratio $B$ indicates the magnitude of $T$-stress relative to the singular term of stress, and its size and sign are dependent on the loading point, crack location and the crack length. Generally speaking, the absolute value of $B$ decreases by moving towards larger $C$ distance.

\section{CONCLUSIONS}

A four-point bending beam is suitable for mixed mode loading just by changing the location of loading point and crack location. The fracture properties, $K_{\mathrm{I}}, K_{\mathrm{II}}$ and $T$-stress expressions for four-point bending were constructed based on pure bending and pure shear by means of finite element calculation. The results reveal that considerable values of the $T$-stress exist with different combinations of $K_{\mathrm{I}}$ and $K_{\mathrm{II}}$, and the stress biaxiality factor $B$ varies, including its magnitude and sign, along with the change in geometry size.

\section{REFERENCES}

[1] Williams, J. and Ewing, P.D. 1972. Fracture under complex stress-the angled crack problem. Internaional Journal of Fracture 8(4): 441-446.

[2] Zhao, Y.H. and Xu, B.H. 2014. Effect of $T$-stress on the mode I fracture toughness of concrete. Comptes rendus-Mécanique, 342(8): 490-500.

[3] Smith, D.J., Ayatollahi, M.R. and Pavierm, M.J. 2001. The role of T-stress in brittle fracture for linear elastic materials under mixed-mode loading. Fatigue and Fracture of Engineering Matererials and Structures 24: 137-150.

[4] Leevers, P.S., Radon, J.C. and Culver, L.E. 1982. Inherent stress biaxiality in various fracture specimen geometries. 
International Journal of Fracture 19: 311325.

[5] Arrea, M. and Ingraffea, A.R. 1981. Mixed-mode crack propagation in mortar and concrete. Department of Structural Engineering, Cornell University, Ithaca, NY, USA, Report 81-31.

[6] Shahani, A.R. and Tabatabaei, S.A. 2008. Computation of mixed mode stress intensity factors in a four-point bend specimen. Appllied Mathematical Modelling 32: 1281-1288.

[7] He, M.Y. and Hutchinson, J.W. 2000. Asymmetric four-point crack specimen. Journal of Appllied Mechanics ASME 67: 207-209.

[8] Ayatollahi, M.R. and Aliha, M.R.M. 2011. On the use of an anti-symmetric fourpoint bend specimen for mode II fracture experiments. Fatigue and Fracture of Engineering Matererials and Structures 34: 898-907.

[9] Hiroshi, T., Paris, P.C. and Irwin, G.R. 2000. The stress analysis of cracks handbook. ASME Press, New York, USA. 